\title{
Useful Plants Grown and Maintained in Domestic Gardens of the Capricorn District, Limpopo Province, South Africa
}

\author{
G. K. E. Mosina ${ }^{1}$, A. Maroyi ${ }^{2 *}$ and M. J. Potgieter ${ }^{3}$ \\ ${ }^{1}$ Department of Microbiology and Plant Pathology Forestry, Agriculture and Biotechnology \\ Institute (FABI), University of Pretoria, Pretoria 0002, South Africa \\ ${ }^{2}$ Department of Botany, University of Fort Hare, Private Bag X1314, Alice 5700, South Africa \\ ${ }^{3}$ Department of Biodiversity, School of Molecular and Life Sciences, University of Limpopo, \\ Private Bag X1106, Sovenga 0727, South Africa
}

KEYWORDS Cultivated Plants. Limpopo Province. Livelihoods. Urbanisation. Useful Plants

\begin{abstract}
The objective of this study was to document useful plants in urban domestic gardens in the Limpopo Province, South Africa. Data on plant use categories of urban domestic gardens in the under-developed areas of the Limpopo Province were collected by means of interviews and personal observations between May and October 2012.A total of 126 taxa belonging to 110 genera and 62 families were recorded from 62 urban domestic gardens. More than half of the species (52.4\%) recorded in the surveyed domestic gardens are exotic to South Africa and 9.5 percent of the total garden flora are "indigenous cultivated", introduced in the Limpopo Province from other provinces of South Africa. The dominant plant use category in domestic gardens was ornamental, followed by fruit trees. The present study revealed that plants grown and maintained in urban domestic gardens play a vital role in the livelihoods of the people of Limpopo Province.
\end{abstract}

\section{INTRODUCTION}

\section{Review of Recent Literature on Domestic Gardens}

Recent studies carried out in Ethiopia (Linger 2014; Mekonnen et al. 2014) and Uganda (Whitney and Gebauer 2014) displayed that domestic gardens can contribute to the development, nutrition and well-being of households managing these agricultural systems. This agro forestry system is believed to be more diverse and, therefore, provide multiple environmental services for households than other monocropping agricultural systems. Other literature on domestic gardens, including Blanckaert et al. (2004), Eichemberg et al. (2009), Maroyi 2013b, Maroyi and Mosina (2014), Molebatsi et al. (2010), Mosina et al. (2014), and Nemudzudzanyi et al. (2010) documented aspects such as food security, income generation, medicinal uses

"Address for correspondence:

Dr. A. Maroyi

Department of Botany,

University of Fort Hare,

Private Bag X1314,

Alice 5700, South Africa

Telehone: +27406022320

Fax: +27866177642

E-mail: amaroyi@ufh.ac.za and ecosystem services that are associated with domestic gardens. From literature, domestic gardens are not only important as sources of food, medicines and income for households involved in their management, but are also important for in-situ conservation of genetic resources for food and agriculture (Agnihotri et al. 2004). The importance of domestic gardens is evident across several countries and societies (Blanckaert et al. 2004; Eichemberg et al. 2009; Molebatsi et al. 2010; Nemudzudzanyi et al. 2010; Maroyi 2013b; Linger 2014; Maroyi and Mosina 2014; Mekonnen et al. 2014; Mosina et al. 2014; Whitney and Gebauer 2014), but there is dearth of information on the importance of urban domestic gardens in South Africa. Thus, the current study was undertaken to investigate plant diversity and uses in urban domestic gardens of the Limpopo Province, South Africa.

A domestic garden is a luxury space around the house used for relaxation, play areas, keeping pets, outdoor eating and cultivation of ornamental plants (Molebatsi et al. 2010). In urban areas flora is important for human well-being and provision of ecosystem services. Urban domestic gardens provide multiple ecosystem services that contribute to quality of life in cities, air quality regulation, carbon capturing (Dunnett and Qasim 2000), temperature regulation (Marco et al. 2010), storm water run-off mitigation 
(Takano et al. 2002), as well as recreational benefits and social cohesion (Wu et al. 2003). Kuruneri-Chitepo and Shackleton (2011) displayed that urban biodiversity enables urban inhabitants to interact with nature; thereby, enhancing appreciation and understanding of the important ecological, social and psychological functions green areas perform. Domestic gardens can also serve as important sources of both food and cash income for vulnerable households in urban areas. The private and public open spaces in cities of the developing world are dotted with gardens producing vegetables, herbs, spices and fruits (Mouget 2006). Private gardening is known to be linked to personal tastes and pleasure (Marco et al. 2010) and which differ according to the garden layout and planted species (Lubbe et al. 2010; Molebatsi et al. 2010). About 22-36 percent of the total urban green space that produces these useful plants, ecosystem goods and services is located in private domestic gardens (Gaston et al. 2005; Mathieu et al. 2007) and probably on 3-4 percent of the total land mass (Alloway 2004; Gibbons et al. 2011; Cameron et al. 2012).

Relatively little has been researched or published on the importance of urban domestic gardens in South Africa. Due to lack of comprehensive data on urban domestic gardens in South Africa, the potential value of urban flora in the provision of food, income and ecosystem services to the fast growing urban population is not clearly known. Previous studies on South African urban domestic gardens focused mainly on plant species distribution, abundance and composition. For instance, McConnachie and Shackleton (2010) and McConnachie et al. (2008) assessed the extent of urban biodiversity across ten small towns in the Eastern Cape Province and found that there was a significant correlation between income levels and public green space provision. The authors found that affluent suburbs had more and diverse public green spaces than poorer ones, an observation also made by Lubbe et al. (2010) based on home gardens in the Tlokwe city municipality in the North West Province. Lubbe et al. (2011) recorded 835 plant species in the Tlokwe city municipality, with 61 percent cultivated for ornamental purposes, 16 percent documented as weeds, 9 percent and 7 percent classified as food and medicinal plants, respectively. Kuruneri-Chitepo and Shackleton (2011) assessed the distribution, composition and abundance of street trees across three towns in the Eastern Cape Province. The authors found noticeable differences in tree density and species richness across suburbs, being highest in the more affluent suburbs and poorly represented in the low income townships. In another study, in the North West Province by Molebatsi et al. (2010), results revealed specific garden layout characterised by six micro-gardens, namely food gardens, medicinal gardens, ornamental gardens, structural species, open and natural areas. Nemudzudzanyi et al. (2009) recorded a total of 149 plant species consisting of 91 medicinal plants, 32 food and 26 spiritual plants in rural and peri-urban domestic gardens in the KwaZulu-Natal Province. Nemudzudzanyi et al. (2009) and Molebatsi et al. (2010) further emphasised the importance of domestic garden plants as sources of food, medicine and structural materials, especially for the poor members of community. All these studies emphasise the potentially significant contribution urban domestic garden flora has to human well-being and urban biodiversity in South Africa. This investigation is part of a larger study (see Mosina et al. 2014) aimed at documenting the ethno botanical knowledge held by local people residing in under-developed areas of the Limpopo Province, South Africa. The current study, therefore, attempts to fill this gap on the knowledge of domestic urban garden flora in the Limpopo Province, South Africa. The study examined the different plant use categories of urban domestic garden plants in the Capricorn District of the Limpopo Province, focusing on both indigenous and alien garden plants.

\section{Objective of the Study}

The objective of this study was to document useful plants in urban domestic gardens in the Limpopo Province, South Africa.

\section{METHODS}

\section{Study Area}

The study was conducted in two towns (Fig. 1) of the Limpopo Province, South Africa. The sites selected for the study were Seshego ( $23^{\circ} 15^{\prime} \mathrm{S} 29^{\circ} 23^{\prime} \mathrm{E}$ ) in Polokwane Municipality and Lebowakgomo (24³1'S2957'E) in LepelleNkumpi Municipality (Fig.1). Seshego is locat- 
ed $13 \mathrm{~km}$ north-west of Polokwane, the capital of the Capricorn District. It is close to the economic core of the district with access to the formal economy of Polokwane Municipality. The township was planned as a dormitory town for workers in Polokwane (Donaldson and Boshoff 2001). Lebowakgomo is located $55 \mathrm{~km}$ south of Polokwane. The main employment sector in Lebowakgomo is the mining industry. The two towns are tertiary or quaternary in nature with 44 percent and 36 percent of the businesses in Lebowakgomo and Seshego respectively being retail shops (Donaldson and Boshoff 2001).

The studied areas are semi-arid, susceptible to frequent droughts and characterised by summer rainfall. Mean annual rainfall ranges from 300 to $500 \mathrm{~mm}$ (LSOER 2004). Daily temperatures vary from mid- $20^{\circ} \mathrm{C}$ to mid- $30^{\circ} \mathrm{C}$, with an average range of between $17^{\circ} \mathrm{C}$ and $27^{\circ} \mathrm{C}$ in summer and $4^{\circ} \mathrm{C}$ to $20^{\circ} \mathrm{C}$ in winter (M'Marete 2003). According to the vegetation classification of $\mathrm{Mu}-$ cina and Rutherford (2006), the Capricorn Dis- trict has a semi-arid savanna, characterised by a mixture of trees, shrubs and grasses. Dominant tree species include Acacia spp., Albizia spp., Combretum spp. and Sclerocarya birrea, with patches of Hyparrhenia spp., Eragrostis spp., Heteropogon spp. and Digitaria spp. grasses.

\section{Research Design and Procedure}

Data on plant use categories in urban domestic gardens in the Limpopo Province were collected by means of semi-structured and structured interviews and personal observations. Thirty one randomly selected individuals from each town were interviewed between May and October 2012.

The interviews were conducted in Sepedi language, since the main author is a native speaker of the language. The aim and purpose of the investigation was explained to the selected participants. The questionnaire used during interviews was designed to gather data on use-

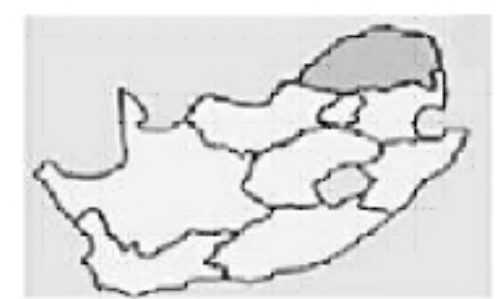

A
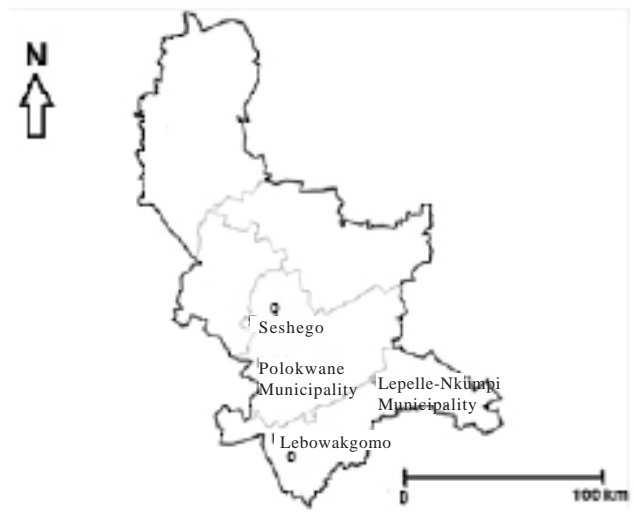

C

Fig.1. A: Geographical location of the study area in South Africa. B: Map of Limpopo Province, showing the geographical position of Capricorn District. C: Detailed map of the study area.

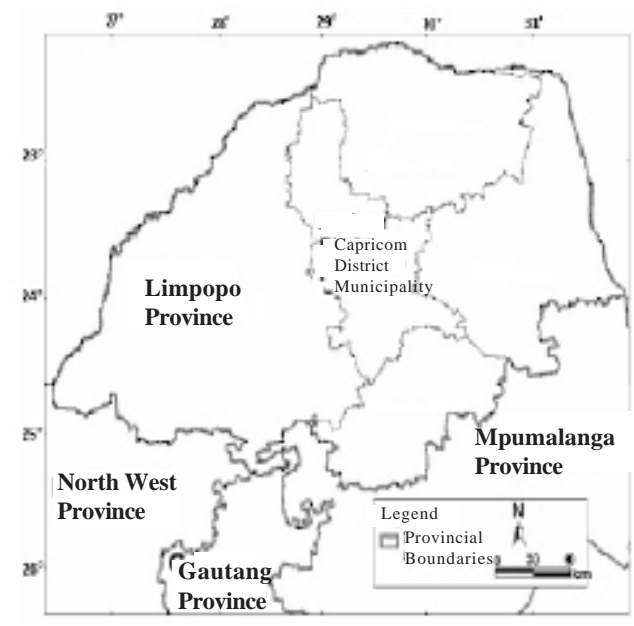

B 
ful plant species (food, medicinal and ornamental) grown and maintained in the domestic gardens. A plant species was included in this study if the home owner could indicate its use. Voucher specimens of plants identified in domestic gardens were collected during the field trips when encountered for the first time and again when they were flowering or fruiting, for easy identification. Each herbarium specimen included important parts such as leaves, stems, flowers and fruits whenever available. For small herbaceous plants, the whole plants were collected. These specimens were deposited for future reference at the Larry Leach Herbarium (UNIN) of the University of Limpopo. The International Plant Name Index (www.ipni.org) and the Royal Botanic Garden and Missouri Botanic Garden plant name database (www.theplantlist.org) were used to validate plant scientific names, plant families and plant authorities.

\section{Analysis of Data}

The data collected were entered in Microsoft Excel 2007 program and were later analysed for descriptive statistical patterns. During analysis, data on useful plants were summarised into major themes by content analysis (Chambers 1994). Descriptive statistics, such as percentages and frequencies were used to analyse the data obtained from the questionnaires. Bar graphs were generated using Microsoft Excel 2007 program.

Species are described as native or alien based on Pyšek et al. (2004). According to Pyšek et al. (2004), naturalised species are defined as aliens that reproduce consistently without direct human intervention, and invasive aliens as naturalised species producing offspring in large numbers and at considerable distances from the parent plants with the potential to spread over a large area. This definition of invasive alien species used in this study is different from the Convention on Biological Diversity (CBD) Conference of Parties' definition of an invasive alien species, where an alien is defined as a species outside its indigenous geographic range, whose introduction and spread threatens biodiversity (UNEP 2002). Another important species classification used in this study is the "indigenous cultivated" category, referring to species indigenous to South Africa and not occurring naturally in the Capricorn District, Limpopo Province, but cultivated in domestic gardens. The origin of "indigenous cultivated" species was determined from Germishuizen et al. (2006). Alien invader species were identified from the national legislation, South Africa 1983, Conservation of Agricultural Resources Act no. 43 (South Africa 1983), and threatened species were identified from the National Red Data List of South Africa's plants (http://redlist.sanbi.org/redcat. php).

\section{Ethical Considerations}

Verbal informal consent was obtained from each individual who participated in the study, and the researchers adhered to the ethical guidelines of the International Society of Ethno biology (www.ethnobiology.net). Interviews were conducted individually whenever possible in an attempt to avoid any direct influences from third parties and to ensure that the data supplied by the participants were as direct and reliable as possible (Phillips and Gentry 1993).

\section{RESULTS}

\section{Species Diversity}

A total of 126 useful taxa belonging to 110 genera and 62 families were recorded from 62 domestic gardens in Lebowakgomo and Seshego in the Limpopo Province (Table 1). Pteridophytes were represented by a single species, Nephrolepis exltata (L.) Schott (family Nephrolepidaceae). Gymnosperms were represented by the Cycadaceae and Zamiaceae families, which in turn consisted of Cycas revoluta Thunb. and Encephalartos transvenosus Stapf and Burtt Davy, respectively. Dicotyledons constituted the majority of the useful plants cultivated in urban gardens in the Limpopo Province, with 95 species (75.4\%) and monocotyledons contributed 28 species (22.2\%). More than half of the species found in the surveyed domestic gardens are exotic to South Africa (52.4\% of the total garden flora), and 9.5 percent of the total garden flora are "indigenous cultivated"; introduced in the Limpopo Province domestic gardens from other provinces of South Africa (Table 1). A large number $(54 \%, n=68)$ of the useful plants in the studied gardens are from 16 families (Fig. 2). The other 46 families had less representation, between 1 to 2 species each. Plant families with the highest number of useful plants were: Asteraceae (10 species), Rosaceae (7 spe- 
Table 1: List of identified plants. Species marked with asterisk $\left(^{*}\right)$ are exotic while those marked with a hatch $(*)$ have been introduced into urban domestic gardens in the Limpopo Province from other provinces in South Africa

\begin{tabular}{|c|c|c|c|c|}
\hline Family, species name & $\begin{array}{l}\text { English } \\
\text { name }\end{array}$ & $\begin{array}{l}\text { Life } \\
\text { form }\end{array}$ & $\begin{array}{c}\text { Use } \\
\text { category }\end{array}$ & $\begin{array}{l}\text { No. of } \\
\text { citations }\end{array}$ \\
\hline \multicolumn{5}{|l|}{ Agapanthaceae } \\
\hline \multicolumn{5}{|l|}{ Hoffmanns ssp africanus } \\
\hline \multicolumn{5}{|l|}{ Agavaceae } \\
\hline $\begin{array}{l}\text { *Agave americana L. } \\
\text { Alliaceae }\end{array}$ & Agave & Shrub & Medicinal & 6.5 \\
\hline "Allium сера L. & Onion & Herb & Vegetable & 16.1 \\
\hline "Allium schoenoprasumL. & Chives & Herb & Vegetable & 3.2 \\
\hline $\begin{array}{l}\text { Tulbaghia violacea Harv. } \\
\text { Amaranthaceae }\end{array}$ & Wild garlic & Herb & Medicinal/ornamental & 4.8 \\
\hline Aerva leucura Moq. & Aerva & Herb & Ornamental & 6.5 \\
\hline Amaranthus hybridus L. & Cape Pigweed & Herb & Vegetable & 22.6 \\
\hline${ }^{*}$ Beta vulgaris L. ssp vulgaris & Beetroot & Herb & Vegetable & 9.7 \\
\hline $\begin{array}{l}\text { "Spinacia oleracea L. } \\
\text { Amaryllidaceae }\end{array}$ & \multicolumn{2}{|c|}{ Amaryllidaceae } & & 29.0 \\
\hline Ammocharis coranica (Ker Gawl.) Herb. & Ground lily & Herb & Medicinal/ornamental & 14.5 \\
\hline $\begin{array}{l}\text { "Clivia miniata (Lindl.) Regel var. miniata } \\
\text { Anacardiaceae }\end{array}$ & Bush lily & Herb & Ornamental & 32.3 \\
\hline Harpephyllum caffrum Bernh. & Wild plum & Tree & Edible fruit & 29.0 \\
\hline${ }^{*}$ Mangifera indica $\mathrm{L}$. & Mango & Tree & Edible fruit & 80.6 \\
\hline *Schinus terebinthifolius Raddi & $\begin{array}{l}\text { Brazilian } \\
\text { pepper tree }\end{array}$ & Tree & Medicinal/ornamental & 48.4 \\
\hline $\begin{array}{l}\text { Sclerocarya birrea (A. Rich.) Hochst. } \\
\text { ssp caffra (Sond.) Kokwaro }\end{array}$ & Marula & Tree & Edible fruit/medicinal & 56.5 \\
\hline $\begin{array}{l}\text { Searsia lancea (L. f.) F. A. Barkley } \\
\text { Anthericaceae }\end{array}$ & Rhus & Tree & Edible fruit/ornamental & 14.5 \\
\hline & \multicolumn{3}{|c|}{ Apiaceae } & 48.4 \\
\hline *Coriandrum sativum $\mathrm{L}$. & Coriander & Herb & Culinary/medicinal & 1.6 \\
\hline \multicolumn{5}{|l|}{ Apocynaceae } \\
\hline *Catharanthus roseus (L.) G. Don. & Periwinkle & Shrub & Medicinal/ornamental & 64.5 \\
\hline \multicolumn{5}{|l|}{ Araceae } \\
\hline *Aglaonema sp & & Herb & Ornamental & 32.3 \\
\hline${ }^{*}$ Monstera deliciosa Liebm. & $\begin{array}{l}\text { Delicious } \\
\text { monster }\end{array}$ & Shrub & Ornamental & 27.4 \\
\hline $\begin{array}{l}\text { Zantedeschia aethiopica (L.) Spreng. } \\
\text { Asphodelaceae }\end{array}$ & Arum lily & Herb & Ornamental & 8.1 \\
\hline $\begin{array}{l}\text { "Haworthia fasciata (Willd.) Haw } \\
\text { Asteraceae }\end{array}$ & \multicolumn{2}{|c|}{ Asteraceae } & Ornamental & 30.6 \\
\hline Artemisia afra Jacq. ex Willd. & $\begin{array}{l}\text { Wild } \\
\text { wormwood }\end{array}$ & Herb & Medicinal & 8.1 \\
\hline \multirow{4}{*}{$\begin{array}{l}{ }^{*} \text { Artemisia vulgaris L. } \\
{ }^{*} \text { Chrysanthemum } s p \\
\text { "Euryops chrysanthemoides (DC.) B. Nord. } \\
\text { Euryops sp }\end{array}$} & \multirow{2}{*}{ Wormwood } & Herb & Medicinal & 11.3 \\
\hline & & Herb & Ornamental & 29.0 \\
\hline & \multirow[t]{2}{*}{ Daisy bush } & Shrub & Ornamental & 51.6 \\
\hline & & Shrub & Ornamental & 32.3 \\
\hline Felicia $s p$ & \multirow[t]{2}{*}{ Bush felicia } & Shrub & Ornamental & 4.8 \\
\hline Gazania $s p$ & & Herb & Ornamental & 40.3 \\
\hline Gerbera jamesonii Adlam & $\begin{array}{l}\text { Barberton } \\
\text { daisy }\end{array}$ & Herb & Ornamental & 29.0 \\
\hline \multirow{2}{*}{$\begin{array}{l}\text { Kleinia longiflora DC. } \\
{ }^{*} \text { Tagetes erecta L. }\end{array}$} & Sjambok bush & Shrub & Medicinal & 8.1 \\
\hline & $\begin{array}{l}\text { Mexican } \\
\text { marigold }\end{array}$ & Shrub & Medicinal & 3.2 \\
\hline \multicolumn{5}{|l|}{ Begoniaceae } \\
\hline $\begin{array}{l}\text { "Begonia homonyma Steud. } \\
\text { Bignoniaceae }\end{array}$ & Wild begonia & Shrub & Ornamental & 45.2 \\
\hline *Tecoma stans (L.) Juss. ex Kunth & Yellow bells & Shrub & Ornamental & 53.2 \\
\hline
\end{tabular}


Table 1: Contd...

\begin{tabular}{|c|c|c|c|c|}
\hline Family, species name & $\begin{array}{c}\text { English } \\
\text { name }\end{array}$ & $\begin{array}{l}\text { Life } \\
\text { form }\end{array}$ & $\begin{array}{l}\text { Use } \\
\text { category }\end{array}$ & $\begin{array}{l}\text { No. of } \\
\text { citations }\end{array}$ \\
\hline \multicolumn{5}{|l|}{ Boraginaceae } \\
\hline${ }^{\#}$ Lobostemon fruticosus (L.) H. Buek & Pajama bush & Shrub & Ornamental & 4.8 \\
\hline \multirow{2}{*}{\multicolumn{5}{|c|}{ 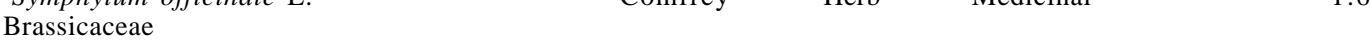 }} \\
\hline & & & & \\
\hline${ }^{*}$ Brassica carinata A. Braun & Cabbage & Herb & Vegetable & 6.5 \\
\hline${ }^{*}$ Brassica juncea (L.) Czern. & Brown mustard & Herb & Vegetable & 29.0 \\
\hline${ }^{*}$ Brassica napus L. & Rape & Herb & Vegetable & 8.1 \\
\hline${ }^{*}$ Brassica rapa L. & Turnip & Herb & Vegetable & 4.8 \\
\hline $\begin{array}{l}{ }^{*} \text { Nasturtium officinale W.T. Aiton } \\
\text { Cactaceae }\end{array}$ & Watercress & Herb & Ornamental & 22.6 \\
\hline $\begin{array}{l}\text { Cactaceae } \\
{ }^{*} \text { Echinopsis spachiana (Lem.) }\end{array}$ & Cactus & Shrub & Medicinal/ornamental & 3.2 \\
\hline \multicolumn{5}{|l|}{ Friedrich and G.D. Rowley } \\
\hline $\begin{array}{l}\text { "Opuntia ficus-indica (L.) Mill. } \\
\text { Caesalpiniaceae }\end{array}$ & Prickly pear & Shrub & Edible fruit/ornamental & 21.0 \\
\hline $\begin{array}{l}\text { Peltophorum africanum Sond. } \\
\text { Cannabaceae }\end{array}$ & Black wattle & Tree & Ornamental & 12.9 \\
\hline $\begin{array}{l}\text { "Cannabis sativa L. } \\
\text { Capparaceae }\end{array}$ & Marijuana & Herb & Medicinal & 6.5 \\
\hline $\begin{array}{l}\text { Cleome gynandra L. } \\
\text { Caricaceae }\end{array}$ & Spider plant & Herb & Ornamental/vegetable & 16.1 \\
\hline $\begin{array}{l}{ }^{*} \text { Carica papaya } \mathrm{L} . \\
\text { Cassuarinaceae }\end{array}$ & Pawpaw & Shrub & Edible fruit/medicinal & 69.4 \\
\hline${ }^{*}$ Cassuarina $s p$ & & Tree & Ornamental & 14.5 \\
\hline \multicolumn{5}{|l|}{ Commelinaceae } \\
\hline Commelina sp & & Herb & Ornamental & 4.8 \\
\hline \multicolumn{5}{|l|}{ Convolvulaceae } \\
\hline $\begin{array}{l}\text { *Ipomoea batatas (L.) Lam. } \\
\text { Crassulaceae }\end{array}$ & Sweet potato & Herb & Edible tuber & 8.1 \\
\hline Cotyledon orbiculata L. & Pig's ear & Shrub & Medicinal/ornamental & 48.4 \\
\hline Crassula capitella Thunb. ssp capitella & Crassula & Shrub & Medicinal/ornamental & 40.3 \\
\hline "Kalanchoe sexagularis N.E.Br. & Kalanchoe & Shrub & Ornamental & 17.7 \\
\hline Kalanchoe sp & Kalanchoe & Shrub & Ornamental & 29.0 \\
\hline Kalanchoe tubiflora (Harvey) Hamet & $\begin{array}{l}\text { Chandelier } \\
\text { plant }\end{array}$ & Shrub & Ornamental & 27.4 \\
\hline \multicolumn{5}{|l|}{ Cucurbitaceae } \\
\hline Citrillus lanatus (Thunb.) Matsum. and Nakai & Watermelon & Herb & Edible fruit & 6.5 \\
\hline Cucurbita pepo L. & Pumpkin & Herb & Edible fruit/vegetable & 12.9 \\
\hline${ }^{*}$ Echevieria $s p$ & & Shrub & Ornamental & 6.5 \\
\hline \multicolumn{5}{|l|}{ Cycadaceae } \\
\hline $\begin{array}{l}\text { *Cycas revoluta Thunb. } \\
\text { Cyperaceae }\end{array}$ & Cycad & Shrub & Ornamental & 4.8 \\
\hline \multirow{2}{*}{\multicolumn{5}{|c|}{ Dracaenaceae }} \\
\hline & & & & \\
\hline Sansevieria hyacinthoides (L.) Druce & $\begin{array}{l}\text { Mother-in-law's } \\
\text { tongue }\end{array}$ & Shrub & Medicinal/ornamental & 45.2 \\
\hline $\begin{array}{l}\text { Euphorbiaceae } \\
\text { Euphorbia } s p\end{array}$ & Euphorbia & Shrub & Ornamental & 4.8 \\
\hline \multicolumn{5}{|l|}{ Geraniaceae } \\
\hline${ }^{\#}$ Pelargonium peltatum (L.) L’Hér & $\begin{array}{l}\text { Ivy-leaved } \\
\text { pelargonium }\end{array}$ & Shrub & Medicinal/ornamental & 30.6 \\
\hline${ }^{\sharp}$ Pelargonium zonale (L.) L’Hér & $\begin{array}{l}\text { Horse-shoe } \\
\text { pelargonium }\end{array}$ & Shrub & Medicinal/ornamental & 40.3 \\
\hline \multicolumn{5}{|l|}{ Hyacinthaceae } \\
\hline Drimia elata Jacq. ex Willd. & & Herb & Medicinal & 4.8 \\
\hline $\begin{array}{l}\text { "Drimiopsis maculata Lindl. and Paxton } \\
\text { Hypoxidaceae }\end{array}$ & Spotted-leaved & Herb & Ornamental & 19.4 \\
\hline $\begin{array}{l}\text { Hypoxis hemerocallidea Fisch., } \\
\text { C.A. Mey. and Avé-Lall }\end{array}$ & Star flower & Herb & Medicinal/ornamental & 4.8 \\
\hline Hypoxis obtusa Burch. ex Ker Gawl. & Star lily & Herb & Medicinal & 1.6 \\
\hline
\end{tabular}


Table 1: Contd...

\begin{tabular}{|c|c|c|c|c|}
\hline Family, species name & $\begin{array}{l}\text { English } \\
\text { name }\end{array}$ & $\begin{array}{l}\text { Life } \\
\text { form }\end{array}$ & $\begin{array}{l}\text { Use } \\
\text { category }\end{array}$ & $\begin{array}{l}\text { No. of } \\
\text { citations }\end{array}$ \\
\hline \multicolumn{5}{|l|}{ Iridaceae } \\
\hline${ }^{\#}$ Dietes grandiflora DC & Large wild iris & Herb & Ornamental & 22.6 \\
\hline $\begin{array}{l}\text { Iris sp } \\
\text { Kirkiaceae }\end{array}$ & Iris & Herb & Ornamental & 1.6 \\
\hline $\begin{array}{l}\text { Kirkia wilmsii Engl. } \\
\text { Lamiaceae }\end{array}$ & \multicolumn{2}{|c|}{ Mountain kirkiaTree } & Medicinal & 1.6 \\
\hline${ }^{*}$ Lavandula angustifolia Mill. & Lavender & Shrub & Culinary/medicinal & 9.7 \\
\hline Mentha longifolia L. & Wild mint & Herb & $\begin{array}{l}\text { Culinary/medicinal/ } \\
\text { ornamental }\end{array}$ & 3.2 \\
\hline Ocimum basilicum L. & Basil & Herb & Culinary/medicinal & 1.6 \\
\hline $\begin{array}{l}{ }^{*} \text { Rosmarinus officinalisL. } \\
\text { Lauraceae }\end{array}$ & Rosemary & Herb & Culinary/medicinal & 3.2 \\
\hline $\begin{array}{l}{ }^{*} \text { Persea americana Mill. } \\
\text { Malvaceae }\end{array}$ & Avocado & Tree & Edible fruit & 66.1 \\
\hline${ }^{*}$ Corchorus olitorius L. var. olitorius & Jute mallow & Shrub & Vegetable & 3.2 \\
\hline $\begin{array}{l}{ }^{*} \text { Hibiscus } s p \\
\text { Mesembryanthemaceae }\end{array}$ & & Herb & Ornamental & 30.6 \\
\hline $\begin{array}{l}\text { Carpobrotus edulis (L.) L. Bolus } \\
\text { Moraceae }\end{array}$ & Hottentot fig & Herb & Medicinal & 29.0 \\
\hline Ficus $s p$ & & Tree & Ornamental & 11.3 \\
\hline "Ficus carica L. & Fig & Tree & Edible fruit & 16.1 \\
\hline $\begin{array}{l}{ }^{*} \text { Morus alba L. } \\
\text { Moringaceae }\end{array}$ & Mulberry & Tree & Edible fruit & 32.3 \\
\hline $\begin{array}{l}{ }^{*} \text { Moringa oleifera Lam. } \\
\text { Musaceae }\end{array}$ & Horseradish & Tree & Medicinal & 11.3 \\
\hline $\begin{array}{l}{ }^{*} \text { Musa } s p \\
\text { Myrothamnaceae }\end{array}$ & Banana & Shrub & Edible fruit & 51.6 \\
\hline Myrothamnus flabellifolius Welw. & $\begin{array}{l}\text { Resurrection } \\
\text { plant }\end{array}$ & Shrub & Medicinal & 6.5 \\
\hline Myrtaceae & & & & \\
\hline $\begin{array}{l}{ }^{*} \text { Psidium guajava L. } \\
\text { Nephrolepidaceae }\end{array}$ & Guava & Tree & Edible fruit & 46.8 \\
\hline $\begin{array}{l}{ }^{*} \text { Nephrolepis exltata (L.) Schott } \\
\text { Nyctaginaceae }\end{array}$ & Maidenhair & Fern & Ornamental & 4.8 \\
\hline $\begin{array}{l}{ }^{*} \text { Bouganvillia sp } \\
\text { Papilionaceae }\end{array}$ & Bouganvillia & Shrub & Ornamental & 41.9 \\
\hline Erythrina lysistemon Hutch. & $\begin{array}{l}\text { Common } \\
\text { coral tree }\end{array}$ & Tree & Medicinal/ornamental & 32.3 \\
\hline Vigna unguiculata (L.) Walp. & Cow pea & Herb & Edible fruit/vegetable & 9.7 \\
\hline Vigna subterranea(L.) Verdc. & $\begin{array}{l}\text { Bambara } \\
\text { groundnut }\end{array}$ & Herb & Edible fruit & 1.6 \\
\hline $\begin{array}{l}\text { Passifloraceae } \\
{ }^{*} \text { Passiflora edulis Sims } \\
\text { Poaceae }\end{array}$ & Granadilla & Shrub & Edible fruit & 8.1 \\
\hline *Saccharum officinarum L. & Sugar cane & Herb & Edible stem & 8.1 \\
\hline "Sorghum bicolor (L.) Moench & Sorghum & Herb & Cereal & 1.6 \\
\hline $\begin{array}{l}{ }^{*} \text { Zea mays L. } \\
\text { Polygalaceae }\end{array}$ & Maize & Herb & Cereal & 12.9 \\
\hline $\begin{array}{l}\text { Securidaca longepedunculata Fresen } \\
\text { Punicaceae }\end{array}$ & Violet tree & Tree & Medicinal/ornamental & 25.8 \\
\hline $\begin{array}{l}{ }^{*} \text { Punica granatum L. } \\
\text { Rosaceae }\end{array}$ & Pomegranate & Tree & Edible fruit & 3.2 \\
\hline *Eriobotrya japonica (Thunb.) Lindl. & Loquat & Tree & Edible fruit & 8.1 \\
\hline${ }^{*}$ Fragaria $x$ ananassa Duchesne & Strawberry & Herb & Edible fruit & 1.6 \\
\hline "Malus domestica Borkh. & Apple & Tree & Edible fruit & 12.9 \\
\hline${ }^{*}$ Prunus armeniaca L. & Apriot & Tree & Edible fruit & 30.6 \\
\hline${ }^{*}$ Prunus persica (L.) Stokes & Peach & Tree & Edible fruit & 72.6 \\
\hline${ }^{*}$ Pyrus communis L. & Pear & Tree & Edible fruit & 8.1 \\
\hline $\begin{array}{l}{ }^{*} \text { Rosa } s p \\
\text { Rubiaceae }\end{array}$ & Rose & Shrub & Ornamental & 30.6 \\
\hline
\end{tabular}


Table 1: Contd...

\begin{tabular}{|c|c|c|c|c|}
\hline Family, species name & $\begin{array}{l}\text { English } \\
\text { name }\end{array}$ & $\begin{array}{l}\text { Life } \\
\text { form }\end{array}$ & $\begin{array}{l}\text { Use } \\
\text { category }\end{array}$ & $\begin{array}{l}\text { No. of } \\
\text { citations }\end{array}$ \\
\hline $\begin{array}{l}\text { Vangueria infausta Burch. ssp infausta } \\
\text { Rutaceae }\end{array}$ & Wild medlar & Shrub & Edible fruit & 1.6 \\
\hline${ }^{*}$ Citrus limon (L.) Burm. f. & Lemon & Shrub & Edible fruit & 54.8 \\
\hline${ }^{*}$ Citrus sinensis (L.) Osbeck & Orange & Shrub & Edible fruit & 51.6 \\
\hline $\begin{array}{l}{ }^{*} \text { Ruta graveolens L. } \\
\text { Sapindaceae }\end{array}$ & Rue & Herb & Medicinal & 1.6 \\
\hline $\begin{array}{l}\text { "Litchi chinensis Sonn. } \\
\text { Solanaceae }\end{array}$ & Litchi & Tree & Edible fruit & 9.7 \\
\hline $\begin{array}{l}\text { "Brunfelsia pauciflora } \\
\text { (Cham. and Schltdl.) Benth. }\end{array}$ & $\begin{array}{l}\text { Yesterday, today } \\
\text { and tomorrow }\end{array}$ & Shrub & Ornamental & 29.0 \\
\hline${ }^{*}$ Capsicum frutescens L. & Chilli & Shrub & Vegetable & 4.8 \\
\hline${ }^{*}$ Lycopersicon esculentum L. & Tomato & Shrub & Vegetable & 14.5 \\
\hline $\begin{array}{l}\text { Petunia sp } \\
\text { Strelitziaceae }\end{array}$ & Leopard tree & Herb & Ornamental & 17.7 \\
\hline${ }^{\#}$ Strelitzia nicolai Regel and Koern. & $\begin{array}{l}\text { Natal wild } \\
\text { banana }\end{array}$ & Tree & Ornamental & 4.8 \\
\hline 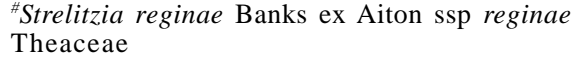 & Bird of paradise & Shrub & Ornamental & 30.6 \\
\hline $\begin{array}{l}{ }^{*} \text { Camellia } s p \\
\text { Verbenaceae }\end{array}$ & & Shrub & Ornamental & 8.1 \\
\hline${ }^{*}$ Duranta erecta L. & Sheenas gold & Shrub & Ornamental & 45.2 \\
\hline $\begin{array}{l}\text { Lippia javanica (Burm. f.) Spreng } \\
\text { Vitaceae }\end{array}$ & Fever tea & Shrub & Medicinal & 9.7 \\
\hline $\begin{array}{l}\text { "Vitis vinifera L. } \\
\text { Xanthorrhoeaceae }\end{array}$ & Grape & Shrub & Edible fruit & 22.6 \\
\hline Aloe ecklonis Salm-Dyck & Grass Aloe & Herb & Ornamental & 1.6 \\
\hline Aloe sp. & Aloe & Herb & Medicinal & 1.6 \\
\hline $\begin{array}{l}\text { "Aloe vera (L.) Burm. f. } \\
\text { Zamiaceae }\end{array}$ & Aloe & Herb & Medicinal/ornamental & 29.0 \\
\hline $\begin{array}{l}\text { Encephalartos transvenosus } \\
\text { Stapf and Burtt Davy }\end{array}$ & Modjadji cycad & Tree & Ornamental & 21.0 \\
\hline
\end{tabular}

cies), Anacardiaceae, Brassicaceae and Crassulaceae (5 species each), Amaranthaceae, Lami-

Table 2: Families with the largest number of useful plants (with more than 3 species) in the Limpopo Province

\begin{tabular}{lcc}
\hline Family & $\begin{array}{l}\text { No. of } \\
\text { species }\end{array}$ & $\%$ \\
\hline Asteraceae & 10 & 7.9 \\
Rosaceae & 7 & 5.6 \\
Anacardiaceae & 5 & 4.0 \\
Brassicaceae & 5 & 4.0 \\
Crassulaceae & 5 & 4.0 \\
Amaranthaceae & 4 & 3.2 \\
Lamiaceae & 4 & 3.2 \\
Solanaceae & 4 & 3.2 \\
Alliaceae & 3 & 2.4 \\
Araceae & 3 & 2.4 \\
Cucurbitaceae & 3 & 2.4 \\
Moraceae & 3 & 2.4 \\
Papilionaceae & 3 & 2.4 \\
Poaceae & 3 & 2.4 \\
Rutaceae & 3 & 2.4 \\
Xanthorrhoeaceae & 3 & 2.4 \\
\hline
\end{tabular}

aceae and Solanaceae (4 species each) and Alliaceae, Araceae, Cucurbitaceae, Moraceae, Papilionaceae, Poaceae, Rutaceae and Xanthorrhoeaceae (3 species each)(Table 2). The genera with the highest number of useful plants were Brassica with four species, followed by Aloe and Kalanchoe with three species each, and Allium, Artemisia, Citrus, Euryops, Ficus, Hypoxis, Pelargonium, Prunus, Strelitzia and Vigna with two species each (Table 1 ).

Seven major uses of domestic garden plants identified in this study (Table 1, Fig. 3) were: Ornamental (44 species), food (41 species), medicinal (16 species), medicinal and ornamental (15 species), food and medicinal (7 species), food and ornamental (3 species) and one species used as food, medicinal and ornamental. The dominant plant use category in domestic gardens was ornamental (Table 1, Fig. 3), followed by fruit trees. Medicinal plants and vegetables were recorded in less than 20\%) of the domestic gardens (Table 1). 


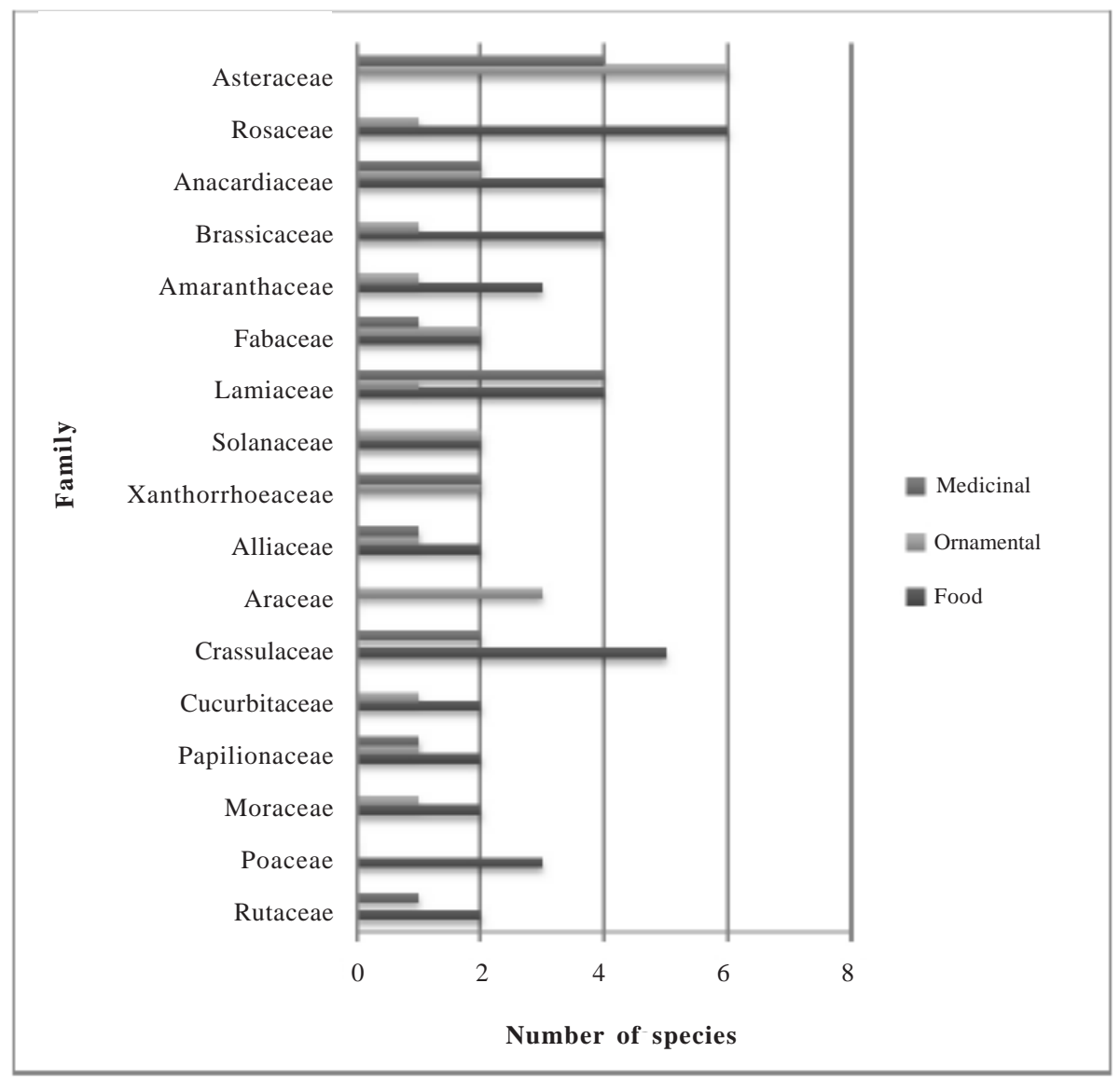

Fig. 2. Families with the largest number of useful plants in the Limpopo Province

\section{Ornamental Plants}

The ornamental plants constituted 49.2 percent of the total urban domestic garden flora in the surveyed gardens with 62 taxa distributed in 42 families. The most important families were: Asteraceae with 6 species, Crassulaceae and Araceae with 5 and 3 species, respectively. Frequent species cultivated and/or maintained by more than 40 percent of the participants included Begonia homonyma, Bouganvillia sp., Chlorophytum comosum, Cyperus sexangularis, Duranta erecta, Euryops chrysanthemoides, Gazania sp. and Tecoma stans. Catharanthus roseus, Cotyledon orbiculata, Crassula capitella subsp capitella, Pelargonium zonale, Sansevieria hyacinthoides, Schinus terebinthifolius occurred in more than 40 percent of the domestic gardens as both ornamental plants and medicines. The majority of plants cultivated and/ or maintained in domestic gardens in the Capricorn District, Limpopo Province as ornamentals are indigenous to South Africa (67.7\%).

The following "indigenous cultivated" species were introduced to the Limpopo Province domestic gardens from other provinces: Begonia homonyma (Eastern Cape, KwaZulu-Natal); Clivia miniata var. miniata (Eastern Cape, KwaZulu-Natal, Mpumalanga); Dietes grandiflora (Eastern Cape, KwaZulu-Natal); Drimiopsis maculata (Eastern Cape, Gauteng, KwaZuluNatal, Mpumalanga); Euryops chrysanthemoides (Eastern Cape, KwaZulu-Natal); Haworthia fasciata (Eastern Cape); Lobostemon fruticosus (Western Cape); Pelargonium peltatum (Eastern Cape, Western Cape ); Pelargonium zonale (Eastern Cape, KwaZulu-Natal, Western Cape); Strelitzia nicolai (Eastern Cape, 


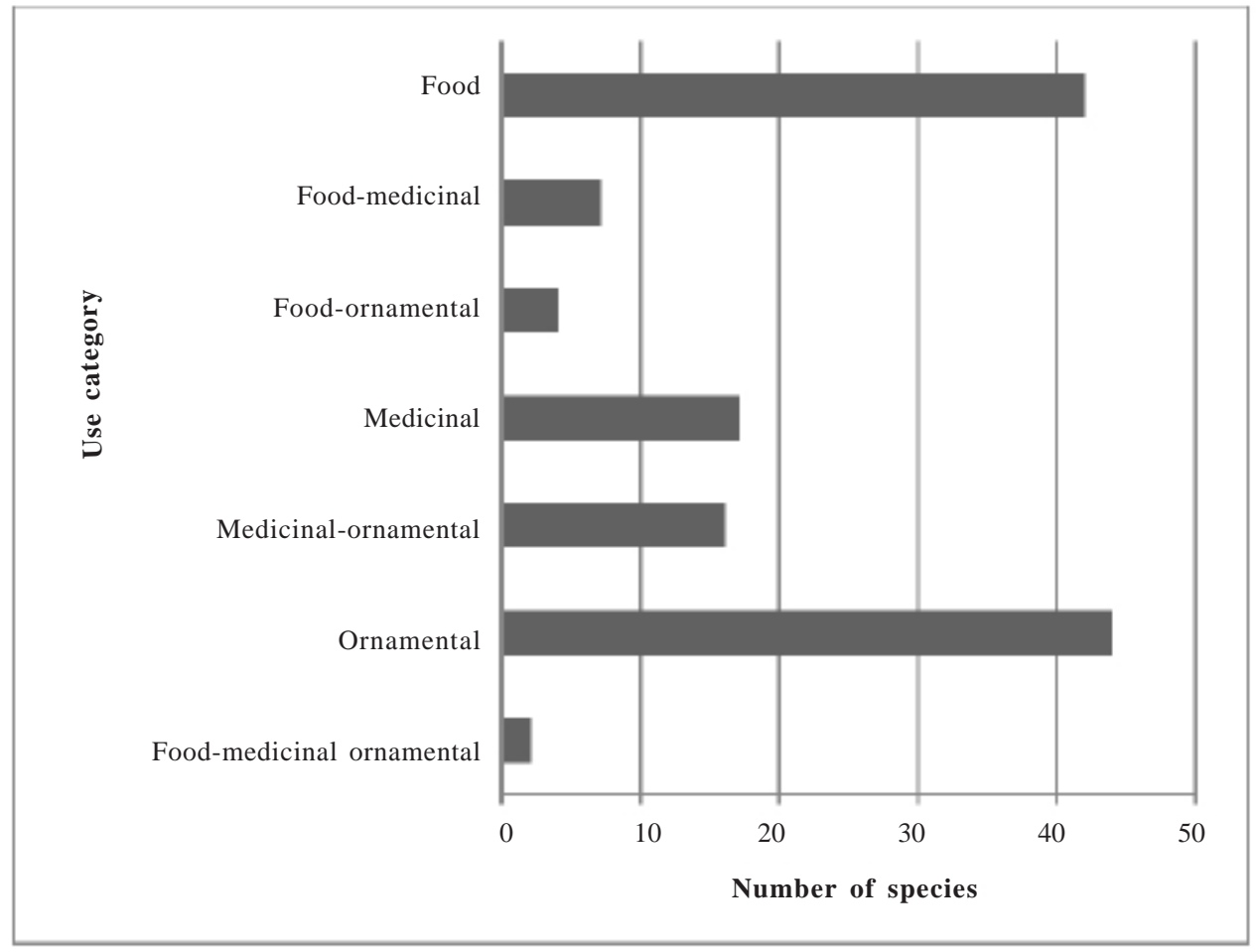

Fig. 3. Number of plants used for food, medicine and as ornamentals in the Limpopo Province

KwaZulu-Natal) and Strelitzia reginae subsp reginae (Eastern Cape, KwaZulu-Natal).

\section{Food Plants}

A variety of food plants were recorded in the studied domestic gardens, mainly fruits (29 species, $23 \%$ ), vegetables (14 species, $11.1 \%$ ), culinary herbs (5 species, 4\%), cereal (2 species) and edible tuber (1 species). Among plants with miscellaneous uses were Saccharum officinarum and Zea mays. Saccharum officinarum was grown in patches in damp places at low elevation for its edible stem and Zea mays was grown for its green corn, which are either roasted or cooked. The most represented families were Rosaceae represented by 6 species; Anacardiaceae, Brassicaceae and Lamiaceae with 4 species each; and Amaranthaceae and Poaceae with 3 species each. The majority of food plants (76.5\%) were exotic. Most food plants (85.2\%) with edible fruits were either trees or shrubs, with Citrillus lanataus, Cucurbita pepo, Fragaria $X$ ananassa, Vigna subterranean and Vigna unguiculata as the only herbaceous plants.

Important exotic fruit trees cultivated and/or maintained by more than 40 percent of the participants included Carica papaya, Citrus limon, Citrus sinensis, Mangifera indica, Musa sp., Persea americana, Prunus persica and Psidium guajava. Indigenous fruit trees cultivated and/or maintained by households included Harpephyllum caffrum, Sclerocarya birrea subsp caffra and Vangueria infausta subsp infausta. Most vegetables (78.6\%) were herbs with Capsicum frutescens, Corchorus olitorius var. olitorius and Lycopersicon esculentum growing as shrubs. All food species from Amaranthaceae and Brassicaceae families were used as vegetables, while members of the Lamiaceae family were used as culinary herbs and/or medicines. 


\section{Medicinal Plants}

Medicinal plants constituted 34 species (27\%) of the total urban domestic garden flora in the Limpopo Province. The most important families were Asteraceae and Lamiaceae represented by four species each. The medicinal plants consisted of mainly herbs (19 species), followed by shrubs (12 species) and four trees. All plants used sorely for medicinal purposes were recorded in less than 29 percent of the domestic gardens. Medicinal plants recorded in more than 30 percent of the domestic gardens were also cultivated and/or managed as ornamentals; and included Catharanthus roseus, Cotyledon orbiculata, Crassula capitella subsp capitella, Erythrina lysistemon, Pelargonium peltatum, Pelargonium zonale, Sansevieria hyacinthoides and Schinus terebinthifolius. Carica papaya and Sclerocarya birrea subsp caffra were the only fruits trees with medicinal uses recorded in more than 30 percent of the domestic gardens.

The following "indigenous cultivated" were introduced to the Limpopo Province domestic gardens from other provinces for their medicinal properties: Agapanthus africanus subsp africanus (Western Cape); Pelargonium peltatum (Eastern Cape, Western Cape); Pelargonium zonale (Eastern Cape, KwaZulu-Natal, Western Cape).

\section{Cultivation of Endemic and Weedy Species in Domestic Gardens}

Agapanthus africanus subsp africanus, endemic to the Western Cape Province was introduced in the Capricorn District domestic gardens for traditional medicine (Table 1). Pelargonium peltatum and Pelargonium zonale were cultivated as ornamentals and for traditional medicine. Most of the endemics (7 species), which included Begonia homonyma, Clivia miniata var. miniata, Dietes grandiflora, Drimiopsis maculata, Euryops chrysanthemoides, Haworthia fasciata, Lobostemon fruticosus and Strelitzia reginae subsp reginae were cultivated as ornamentals.

About 10 percent (13 species) of the total garden flora recorded in this study are declared weeds and invaders in South Africa, listed under the Conservation of Agricultural Resources Act (1983) No. 43 of 1983. Among them were: Agave americana, Catharanthus roseus, Duran- ta erecta, Echinopsis spachiana, Eriobotrya japonica, Morus alba, Nasturtium officinale, Nephrolepis exltata, Opuntia ficus-indica, Passiflora edulis, Psidium guajava, Schinus terebinthifolius and Tecoma stans.

\section{DISCUSSION}

\section{Cultivation and Maintenance of Plants in Domestic Gardens}

The results obtained in this study corroborate those from other countries, demonstrating that urban dwellers are actively engaged in planting and maintenance of plant species in domestic gardens, a fact often overlooked in debates around urban planning. For example, Linger (2014) and Mekonnen et al. (2014) argued that domestic gardens in Ethiopia are diverse and therefore able to provide multiple goods and ecosystem services to households than monocropping agro forestry systems. Whitney and Gebauer (2014) found domestic gardens in Uganda to be sustainable small-scale agro forestry systems important as sources of food, income and medicines as well as important for conservation of biodiversity. Results obtained in the current investigation showed a range of uses, indicating that garden owners have a broad knowledge of plants and their uses. Across the two towns, the predominant use of cultivated and maintained plants in domestic gardens is ornamental purpose. The term ornamental is here used in a wide and general sense including all decorative uses, that is, hedge and house plants. Based on these results, it can be inferred that species diversity grown and maintained in domestic gardens in the Capricorn District play a major aesthetic and decoration function. Nair (1993) explored that the high number of ornamental plants in urban gardens is associated with the aesthetic role of domestic gardens in cities, since they are not used for subsistence, except among low income city dwellers. Similarly, Reichard and White (2001) showed that large number of plant species introduced into the urban environment are for horticultural purposes.

The dominance of ornamental plants in the Capricorn District urban gardens implies that food plants in these gardens play a supplementary role. These results are comparable to studies done by Blanckaert et al. (2004), who found ornamental plants dominating the plants culti- 
vated in home gardens of San Rafael Coxcatlan, valley of Tehuacan-Cuicatlan, Mexico, representing as much as 65.7 percent of the total garden flora, while edible plants and medicinal were 29.6 percent and 8.6 percent, respectively. In a survey conducted in Rio Claro, southeast Brazil, of 410 species documented in urban home gardens, 257 species were cultivated for ornamental purposes, 98 species for food and 93 species for medicinal uses (Eichemberg et al. 2009). In peri-urban domestic gardens of the North West Province, South Africa, Molebatsi et al. (2010) recorded 60 percent ornamental, 21 percent food and 12 percent medicinal plants. In another study, in the North West Province, Lubbe et al. (2011) recorded 61 percent ornamental, 16 percent weedy species, 9 percent food and 7 percent medicinal plants. The number of ornamental plants is said to be high in Tlokwe Municipality, North West Province because of large variety of plants that are available to the home gardener from nurseries and also nurseries promoting planting of ornamentals (Lubbe et al. 2011). The emphasis of urban home gardening appears not to be food or traditional medicine production as is the case in rural domestic gardens (Kumar and Nair 2004). But urban dwellers manage plant species to meet diverse livelihood needs, including the enhancement of human well-being given the social benefits and recreational opportunities that garden plants offer to inhabitants (Kuruneri-Chitepo and Shackleton 2011). Further, it was found that although food plants were cultivated and maintained, people actively planted fruit trees such as Carica papaya, Citrus limon, Citrus sinensis, Mangifera indica, Musa sp, Persea americana, Prunus persica and Psidium guajava. Only two cereal and fourteen vegetable species (Table 1) were cultivated and maintained in the Capricorn District urban gardens. Medicinal plants made very small contributions to the diversity of the garden flora in the Capricorn District. A low representation of medicinal plants in urban gardens was also noted in Brazil (Blankaert et al. 2004) and the North West Province of South Africa (Lubbe et al. 2011; Molebasti et al. 2010).

\section{Cultivation of Weedy and Endemic Species in Domestic Gardens}

Exotic and invasive plant species are widely cultivated in the Limpopo Province urban gar- dens, among them are: Agave americana, $\mathrm{Ca}$ tharanthus roseus, Duranta erecta, Echinopsis spachiana, Eriobotrya japonica, Morus alba, Nasturtium officinale, Nephrolepis exltata, Opuntia ficus-indica, Passiflora edulis, Psidium guajava, Schinus terebinthifolius and Tecoma stans. The majority of these species pose an immediate and significant threat by virtue of their aggressive qualities and having the capacity to invade natural habitats and overwhelm some of the indigenous species (South Africa 1983). Due to the ecological effect invasive species have on the environment with regard to their serious health risk to humans or livestock, causing serious financial losses to land users, their ability to invade undisturbed environments and transform or degrade natural plant communities, use more water than the plant communities they replace or be particularly difficult to control, Regulation 15, Act No. 43 of 1983 was enacted. Second to habitat destruction and modification, alien invasion is recognised as having the largest impact on natural vegetation, ecosystem processes and interfering with agricultural practices (Heywood 1995; Mooney and Hobbs 2000; Binns et al. 2001; Bigirimana et al. 2011, 2012). The Capricorn District urban gardens harbour 66 exotic species (52.4\% of the total garden flora) that could escape from the gardens and naturalise. Similarly, Lubbe et al. (2011) recorded 88 declared invader and weedy species in the Tlokwe city municipality, the North West Province. In South Africa, at least 161 species cause serious problems in natural and semi-natural ecosystems (Henderson 1995), impacting on approximately 8.6 percent of the country's total land surface or roughly 10 million hectares (Le Maitre et al. 2000). This is because the cities act as immigration sources from which the alien species can disperse into the surrounding landscape (McConnachie et al. 2008; Bigirimana et al. 2011, 2012). During 2005/ 2006 financial year, R3.2 billion was spent on clearing alien vegetation on 1.6 million hectares of land (Marais and Wannenburgh 2008). This cost is further compounded by follow up clearing programs (Maraiset al.2004). Studies by Zimmermann and Neser (1999), Stepp and Moerman (2001), Njoroge et al. (2004), Bigirimana et al. (2011, 2012), Semenya et al. (2012) andMaroyi (2013a) showed that invasive plants may also have positive economical, social and ecological significance and these need to be taken into account when assessing the costs resulting from invasions. 
Five invasive species documented in this study: Agave americana (medicinal), Catharanthus roseus (medicinal/ornamental), Eriobotrya japonicaand Psidium guajava (edible fruits) and Opuntia ficus-indica (edible fruit/ornamental) are all used as herbal medicines by Bapedi traditional healers in the Limpopo Province (Semenya et al. 2012; Semenya and Potgieter 2014). Similarly, Dold and Cocks (2002), noted that of the 130 plant species used as traditional medicine by Xhosa traditional healers in the Eastern Cape Province, 33 are declared exotic species. Opuntia ficus-indica and Psidium guajava are widely cultivated for fruit production in South Africa (Zimmermann and Neser 1999) and Zimbabwe (Maroyi 2013b). There is now a large body of evidence supporting human dependency on invasive alien plant species for food, shelter, ecosystem services, aesthetic enjoyment and cultural identity (Zimmermann and Neser 1999). The results of this study, therefore, calls for a review of the socio-economic benefits of exotic plants to local communities in South Africa before blindly advocating for their eradication. In addition, the extensive use of exotic plants is seen as imperative for their ultimate control and should ultimately form part of their management strategy (Semenya et al. 2012). As part of this management strategy, garden owners should be educated on the management of some of the invasive species, especially those listed in category 1 of the Conservation of Agricultural Resources Act (1983) No. 43 of 1983.

The researchers noted, like in a previous study by Lubbe et al. (2011), a high proportion of "indigenous cultivated" species $(9.5 \%, n=12)$ in our studied urban gardens in the Capricorn District. All these species with the exclusion of Strelitzia nicolai are endemics. Agapanthus africanus subsp africanus was introduced because of its medicinal properties (Table 1), while Pelargonium peltatum and Pelargonium zonale were introduced for medicinal and ornamental uses. Begonia homonyma, Clivia miniata var. miniata, Dietes grandiflora, Drimiopsis maculata, Euryops chrysanthemoides, Haworthia fasciata, Lobostemon fruticosus, Strelitzia nicolai and Strelitzia reginae subsp reginae were cultivated for ornamental purposes. The presence of these species from other provinces may point out to the possibility of exchange and sharing of ethno botanical information concerning particularly traditional medicines. Research by Maroyi (2013a) showed that household owners give home garden products to neighbours and relatives, and this exchange between households and relatives strengthen relationships.

Further, domestic gardens can play a unique role by contributing to livelihood needs of households, selection and distribution of species, as well as conservation of endemic or economically valuable species. Management of plant diversity in the domestic gardens in this way ensures their availability to the present as well as future generations and this is a combination of ex situ and circa situ conservation (Hamilton 2004). This conservation initiative appears not to be planned and we doubt if the owners of the domestic gardens are aware that some of the cultivated species are endemics. Species listed in the South African Red Data List benefiting from this initiative are Begonia homonyma, Clivia miniata var. miniata, Haworthia fasciata and Hypoxis hemerocallidea. All these species are cultivated in the Capricorn District for ornamental purposes with the exclusion of Hypoxis hemerocallidea cultivated for medicinal purposes (Maroyi and Mosina 2014). But conservation assessments made by (Raimondo et al. 2009) revealed that over-exploitation of these species as traditional medicines and habitat loss are major causes of threats. Begonia homonyma (EN C2a(i) is generally rare and over-exploited for traditional medicine. Clivia miniata var. miniata (VU A2abcd), is threatened by harvesting for the traditional medicine trade. Haworthia fasciata (NT B1ab(ii,iii,iv,v),is threatened by habitat loss due to urban expansion around Port Elizabeth, as well as ploughing for pasture and agricultural expansion around Humansdorp in the Eastern Cape Province. Hypoxis hemerocallidea is listed as declining (Raimondo et al. 2009). However, the species is heavily harvested for the medicinal plant trade throughout the country and is also threatened by land transformation and habitat loss in Gauteng Province. There is a need, therefore, to encourage households to manage these species in domestic gardens, before their medicinal value vanishes as they disappear through over-exploitation and habitat loss in South Africa.

\section{CONCLUSION}

The study revealed that plants grown and maintained in domestic gardens play a vital role 
in the livelihoods of the people of the Limpopo Province, South Africa. They are important as ornamental, food and medicines. Although the majority of these species are exotic and some "indigenous cultivated", these species have become imbedded in the lives and cultures of the people of the Limpopo Province. These findings with respect to the importance of urban domestic gardens and their preferences for certain species and use categories, has implications for policy regarding the planting and management of plants in urban domestic gardens. It is difficult for public authorities to influence the management of private gardens but local authorities can educate communities in urban centres on some of these aspects. The role and value of plants to urban livelihoods should be taken into account in planning by the relevant municipal and government agencies. Moreover, maintenance of green spaces and trees within urban areas is now widely recognised as one of the primary strategies available to urban planners to contribute to urban ecology and regular human contact with nature for their physical and psychological well-being. It is recommended that urban households be made aware of the extensive variety of goods and services trees provide and the important role plants can play in helping to sustain urban livelihoods.

\section{RECOMMENDATIONS}

The potential value of urban domestic gardens in the provision of goods and environmental services to the highly growing urban population in South Africa is not known, due to lack of comprehensive data on urban domestic garden flora. This study demonstrated that urban domestic gardens in the Limpopo Province, South Africa are of potential significance to biodiversity conservation, ecosystem services and the well-being of the local communities as sources of food and medicinal plants. Documentation of garden flora is, therefore, a critical starting point in trying to understand the importance of plant biodiversity in urban domestic gardens to the livelihoods and provision of ecosystem services to local communities. There is need therefore, to carry out similar studies in other provinces of South Africa to enhance appreciation and understanding of the ecological and social importance of urban domestic garden flora.

\section{ACKNOWLEDGEMENTS}

We are indebted to the National Research Foundation, South Africa for funding this research. Our sincere gratitude goes to the friendly people of Lebowakgomo and Seshego for allowing us into their gardens.

\section{REFERENCES}

Agnihotri RK, Sharma S, Joshi M, Paini LMS 2004. Crop diversity in home gardens of Kumaun region, central Himalaya, India. Pl Genetic Res, 138: 2328.

Alloway BJ 2004. Contamination of soils in domestic gardens and allotments: A brief overview. Land Contam Reclamat, 12: 179-187.

Bigirimana J, Bogaert J, De Canniere C, Bigendako MJ, Parmentier I 2012. Domestic garden plant diversity in Bujumbura, Burundi: Role of the socio-economical status of the neighbourhood and alien species invasion risk. Landscape Urb Plan, 107: 118126.

Bigirimana J, Bogaert J, De Canniere C, Lejoly J, Parmentier I 2011. Alien plant species dominate the vegetation in a city of sub-Saharan Africa. Landscape Urb Plan, 100: 251-267.

Binns JA, Illgner PM, Nel EL 2001. Water shortage, deforestation and development: South Africa's working for water programme. Land Degrad Devel, 12: 341-355.

Blanckaert I, Swennen RL, Flores PM, Lopez RI, Saade RL 2004. Floristic composition, plant uses and management practices in home gardens of San Rafael Coxcatlan, valley of Tehuacan-Cuicatlan, Mexico. $J$ Arid Env, 57: 39-62.

Cameron RWF, Blanmsa T, Taylor JE, Salisbury A, Halstead AJ, Henricot B, Thompson K 2012. The domestic garden: Its contribution to urban green infrastructure. Urb For Urb Green, 11: 129-137.

Chambers R 1994. The origins and practice of participatory rural appraisal. World Devel, 22: 953-969.

Dold AL, Cocks ML 2002. The trade in medicinal plants in the Eastern Cape Province, South Africa. S Afr J Sci, 98: 589-597.

Donaldson SE, Boshoff E 2001. Household linkages in dispersed settlement around Pietersburg and implication for household resources management. J Fam Ecol Consumer Sci, 29: 20-27.

Dunnett N, Qasim M 2000. Perceived benefits to human well-being of urban gardens. Hort Techn, 10: 40-45.

Eichemberg MT, Amorozo MC, De MouraLC 2009. Species composition and plant use in old urban homegardens in Rio Claro, southeast Brazil. Acta Bot Bras, 23: 1057-1075.

Germishuizen G, Meyer NL, Steenkamp Y, Keith M 2006. A Checklist of South African Plants. Southern African Botanical Diversity Network Report No. 41, SABONET, Pretoria, South Africa.

Gibbons S, Mourato S, Resende G 2011. The Amenity Value of English Nature: A Hedonic Price Approach. Spatial Economics Research Centre (SERC). Lon- 
don, UK: London School of Economics and Political Sciences.

Hamilton AC 2004. Medicinal plants, conservation and livelihoods. Biod Cons,13: 1477-1517.

Henderson L 1995. Plant Invaders of Southern Africa.Plant Protection Research Institute Handbook No. 5.Pretoria, South Africa: Agricultural Research Council.

Heywood VH 1995. Global Biodiversity Assessment. Cambridge, UK: Cambridge University Press.

Kumar BM, Nair PKR 2004. The enigma of tropical home gardens. Agrofor Syst, 61: 135-152.

Kuruneri-Chitepo C,Shackleton CM 2011. The distribution, abundance and composition of street trees in selected towns of the Eastern Cape, South Africa. Urb For Urb Green, 10: 247-254.

Le Maitre DC, Versfeld DB, Chapman RA 2000. The impact of invading alien plants on surface water resources in South Africa: A preliminary assessment. Water SA, 26: 397-408.

Limpopo State of the Environment Report (LSOER) 2005. State of the Environment Reports. From <http: www.environment.gov.za.> (Retrieved on 18 May 2013).

Linger E 2014. Agro-ecosystem and socio-economic role ofhomegarden agro-forestry in Jabithenan District, north-western Ethiopia: Implication for climate change adaptation. SpringerPlus, 3: 154.

Lubbe CS, Siebert SJ, Cilliers SS 2010. Political legacy of South Africa affects the plant diversity patterns of urban domestic gardens along a socio-economic gradient. Sci Res Essays,5: 2900-2910.

Lubbe CS, Siebert SJ, Cilliers SS 2011. Floristic analysis of domestic gardens in the Tlokwe City Municipality, South Africa. Bothalia, 41: 351-361.

Marais C, Wannenburgh AM 2008. Restoration of water resources (natural capital) through the clearing of invasive alien plants from riparian areas in South Africa: Costs and water benefits. S Afr J Bot, 74: 526-537.

Marais C, Van Wilgen BW, Stevens D 2004. The clearing of invasive alien plants in South Africa: A preliminary assessment of costs and progress. S Afr $J$ Sci, 100: 97-103.

Marco A, Lavergne S, Du Toit T, Bertaudiere-Montes V 2010. From the backyard to the back country: How ecological and biological traits explain the escape of garden plants into Mediterranean old fields. Biol Invas, 12: 761-779.

Maroyi A 2013a. Use of weeds as traditional vegetables in Shurugwi District, Zimbabwe. J Ethnobiol Ethnomed, 9: 60.

Maroyi A 2013b. Use and management of homegarden plants in Zvishavane District, Zimbabwe. Trop Ecol, 54: 191-203.

Maroyi A, Mosina GKE 2014. Medicinal plants and traditional practices in peri-urban domestic gardens of the Limpopo Province, South Africa. Indian J Indig Knowl, 13: 665-672.

Mathieu R, Freeman C, Aryal J 2007. Mapping private gardens in urban areas using object-oriented techniques and very high-resolution satellite imagery. Landscape Urb Plan, 81: 179-192.

McConnachie MM, Shackleton CM 2010. Public green space inequality in small towns in South Africa. Habitat Int, 34: 244-248.
McConnachie MM, Shackleton CM, McGregor GK 2008. The extent of public green space and alien plant species in 10 small towns of the sub-tropical thicket biome, South Africa. Urb For Urb Green, 7: $1-13$.

Mekonnen EL, Asfaw Z, Zewudie S 2014. Plant species diversity of homegarden agroforestry in Jabithenan District, north-western Ethiopia. Int J Biod Cons, 6: 301-307.

M'marete CK 2003. Climate and Water Resources in the Limpopo Province. Polokwane, South Africa: Limpopo Department of Agriculture.

Molebatsi LY, Siebert SJ, Cilliers SS, Lubbe CS, Davoren NE 2010. The Tswana tshimo: A home garden system of useful plants, with a specific layout and function. Afr J Agr Res, 5: 2952-2963.

Mooney HA, Hobbs RJ 2000. Invasive Species in a Changing World. Washington DC, USA: Island Press.

Mosina GKE, Maroyi A, Potgieter MJ 2014. Comparative analysis of plant use in peri-urban domestic gardens of the Limpopo Province, South Africa. $J$ Ethnobiol Ethnomed,10: 35.

Mouget LJA 2006. Growing Better Cities: Urban Agriculture for Sustainable Development. Ottawa, Canada: International Development Research Centre (IDRC).

Mucina L, Rutherford MC 2006. The Vegetation of South Africa, Lesotho and Swaziland. Pretoria, South Africa: South African National Biodiversity Institute. Strelizia 19.

Nair PKR 1993. An Introduction to Agro-forestry. London, UK: ICRAF/KLUWER, Academic Publishers.

Nemudzudzanyi AO, Siebert SJ, Zobolo AM, Molebatsi LY 2010. The Zulu muzi: A homegarden system of useful plants with a specific layout and function. Afr J Indig Knowl Syst, 9: 57-72.

Njoroge NG, Bussmann WR, Gemmill B, Newton LE, Ngumi VW 2004. Utilisation of weed species as sources of traditional medicines in central Kenya. Lyonia, 7: 71-87.

Phillips OLB, Gentry AH 1993. The useful plants of Tambopata, Peru: I. Statistical hypothesis tests with a new quantitative technique. Econ Bot, 47: 15-32.

Pyšek P, Richardson DM, Rejmanek M, Webster GL, Williamson M, Kirschner J 2004. Alien plants in checklists and flora: Towards better communication between taxonomists and ecologists. Taxon, 53: 131143.

Raimondo D, Von Staden L, Foden W, Victor JE, Helme NA, Turner RC, Kamundi DA, Manyama PA 2009. Red List of South African Plants. Pretoria, South Africa: South African National Biodiversity Institute. Strelitzia 25.

Reichard SH, White P 2001. Horticultural introductions of invasive plant species: A North American perspective. In: JA McNeely (Ed.): The Great Reshuffling: Human Dimensions of Invasive Alien Species. Gland, Switzerland: IUCN, pp. 161-170.

Semenya SS, Potgieter MJ 2014. Bapedi traditional healers inthe Limpopo Province, South Africa: Their socio-cultural profile and traditional healing practice. J Ethnobiol Ethnomed, 10: 4.

Semenya SS, Potgieter MJ, Tshisikhawe MP, Shava S, Maroyi A 2012. Medicinal utilization of exotic plants by Bapedi traditional healers to treat human ail- 
ments in Limpopo Province, South Africa. J Ethnopharmacol, 144: 646-655.

South Africa 1983. Conservation of Agricultural Resources Act No. 43 of 1983. Pretoria, South Africa: Government Printer.

Stepp JR, Moerman DL 2001. The importance of weeds in ethnopharmacology. J Ethnopharmacol, 75: 1923.

Takano T, Nakamura K, Watanabe M 2002. Urban residential environments and senior citizens' longevity in megacity areas: the importance of walkable green spaces. J Epidemiol Comm Health, 56: 913-918.

UNEP (United Nations Environmental Programme) 2002. Alien Species that Threaten Ecosystems, Hab- itats or Species. From <http://www.cbd.int.> (Retrieved on 21 July 2013).

Whitney CW, Gebauer J 2014. Homegardens in Uganda: Diversity and Potential. In: G Rahmann, U Aksoy (Eds.): Building Organic Bridges. Proceedings of the $4^{\text {th }}$ ISOFAR Scientific Conference, Organic World Congress, Istanbul, Turkey, 13-15 October 2014.

Wu J, Jenerette GD, David J 2003. Linking land-use change with ecosystem processes: A hierarchical patch dynamic model. In: S Guhathakurta (Ed.): Integrated Land Use and Environmental Models. Berlin, Germany: Springer, pp. 99-119.

Zimmermann HG, Neser S 1999. Trends and prospects for biological control of weeds in South Africa. Afr Entomol Mem, 1: 165-173. 